\title{
Metal Chelation in Asymmetric Diels-Alder Reaction (II)
}

\author{
Kyoo-Hyun Chung, ${ }^{*}$ Chan-Kook Chu, and Min-Ho Chang \\ Department of Chemistry. Inha University. Inchon 402-751, Korea \\ Received November 17.2003
}

Key Words : Metal chelation, Lewis acid, Asymmetric synthesis, Diels-Alder reaction

Diels- $\Lambda$ der reaction is one of the most effective methods to prepare complex organic molecules.' The reaction provides up to four stercogenic centers in one slep. The asymmetric Diels- $\Lambda$ lder reaction has been carried out using a chiral auxiliary and a chiral catalys $1^{2.3}$ Chiral dienophiles were olten employed as chiral auxiliaries, derived from many functional groups such as alcohols, amines, oxazolidinones, and sultams. ${ }^{+7}$ Diels- $\wedge$ der cycloadditions of chiral dienophiles with cyclopentadiene proceed with diastereofacial sclectivity, giving either endo-R or endo-S compounds as a major component, depending on Lewis acids used.

In the reaction, the inverse asymmetric induction has been observed by the use of either monovalent Lewis acids or divalent Lewis acids. The reaction of the acrylate of (S)-ethyl lactate with eyclopentadiene proceeded with $86 \%$ endo-S selectivity in the presence of $\mathrm{TiCl}_{4}$, divalent Lewis acid, whereas did with $32 \%$ ende-R selectivity in the presence of $\mathrm{BF}_{3} \cdot \mathrm{El}_{2} \mathrm{O}$, monovalent Lewis acid. ${ }^{5}$ When a chiral dienophile which was prepared from benzyl ester of $(S)$-proline ${ }^{6}$ or methyl ester of (S)-indoline-2-carboxylic acid ${ }^{7}$ was employed in the reaction, a similar diastereofacial selectivity was also observed. Recently, we reported the similar selecivity in the reaction of methyl (2S.5S)-(5-t-butyldimethylsilyloxypiperidin-2-yl)ethanoate with cyclopentadiene.

The diflerent diastercoselectivity in the reaction was often rationalized by the chelation between dienophiles and Lewis acids. ${ }^{5-8}$ The coordination between a metal and a substrate was proven by $\mathrm{X}$-Ray analysis; 5-membered ring complex was formed between $\mathrm{TiCl}_{4}$ and ethyl lactate. In the ease of $\alpha, \beta$-unsaturated $N$-acyloxazolidinones, 6-membered chelation was proposed by a NMR spectral analysis. ${ }^{10}$ According to Waldmann's" and Kim's reports, 7-membered ring complex was formed between $\mathrm{TiCl}_{4}$ and dienophiles. We also suggested that 8-membered chelation might affect the diastereoselectivity.

In this report, we tried to elucidate the effect of 8membered ring complex in Diels-Alder reaction. Axial ester 3, prepared from methyl 8-aza-3 $\beta$-benzoyloxybicyclo[3.2.1] octane-2 $\beta$-carboxylate, might be able to form 8membered chelation between axial ester 3 and $\mathrm{TiCl}_{4}$, a divalent Lewis acid catalyst. Whereas equatorial ester $\mathbf{5}$ should not form any cyclic complex with $N$-acyl moiety.

As shown in Table I, the amount of $\mathbf{4 a}$ and $\mathbf{4 b}$ was almost same when the reaction was taken place without a catalyst (entry 1). In the presence of monovalent Lewis acids, $4 \mathbf{a}$ was given as a major compound in the reaction of 3 (entries 2,3). However, divalent Lewis acids were employed in the reaction, $\mathbf{4 b}$ was major (entries 4,5 ). In the reaction of dienophile 3, a best diastereoselectivity was obtained with $\mathrm{AlC}^{2}$ : (entry 2). Again as shown in Table 2, without a catalyst, the amount of $\mathbf{6 a}$ and $\mathbf{6 b}$ was almost same in the reaction of 5 , the $\mathrm{C} 2$ epimer of 3 (entry 1). However, the reaction of dienophile 5 with cyclopentadiene proceeded with same diastereoselectivity to give $\mathbf{6 a}$ as a major

Table 1. Asymmetric Diels- $A$ kder Reaction of 3 with cyclopentadiene

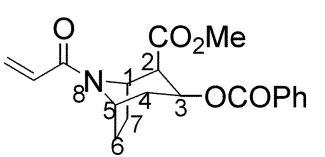

3

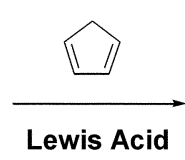

Lewis Acid

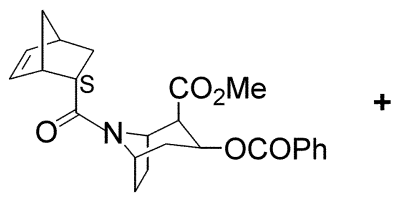

$4 a$

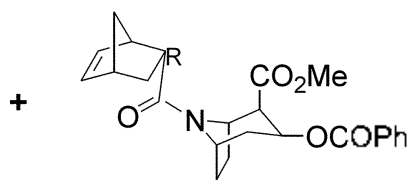

$4 b$

\begin{tabular}{|c|c|c|c|c|c|c|c|}
\hline lintry & I enz is acid & lemp ( ${ }^{\circ}(\mathrm{C})$ & T"iוise (h) & Yield $(\%)$ & Endo: Exo" & findo ds" & Conlige' \\
\hline I & none & RT & 72 & 30 & $70: 30$ & 1.5 & $\mathrm{~S}$ \\
\hline 2 & $\triangle I C]_{3}$ & 0 & 15 & 88 & $78: 22$ & 99 & $\mathrm{~S}$ \\
\hline 4 & $\mathrm{TiCl}_{4}$ & 0 & 16 & 80 & $63: 37$ & 73 & $\mathrm{R}$ \\
\hline 5 & $\mathrm{Ti}\left(\mathrm{OPr}^{\prime}\right)_{\mathrm{d}}$ & RT & 24 & 74 & $71: 29$ & 80 & $\mathrm{R}$ \\
\hline
\end{tabular}

"The ratio of the amount of $4 \mathrm{a}$ and $\mathbf{t b}$ to their corresponding exo isomers. "Calculated by the amounts of $4 \mathrm{a}$ and $\mathbf{t b}$. The chirality of $\mathrm{C} 2 \mathrm{2}$ position in the norbonene moiety of the major compound. The authentic 4 b was prepared from endo-(2R)-5-norbonene-2-carboxylic acid."

\footnotetext{
"Corresponding Author. e-mail: kyoohyun( $a$ inha.ac.kr
} 
Table 2. Asymmetric Diels- $\wedge$ lder Reaction of 5 with cyclopentadiene

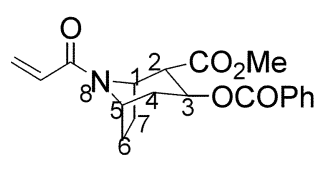

5

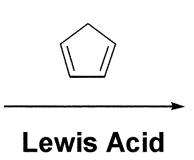

Lewis Acid

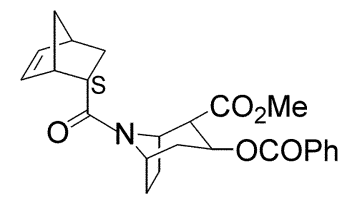

6 a

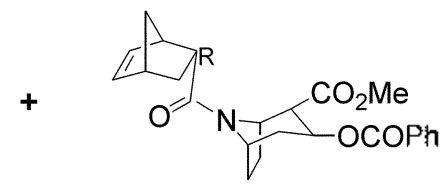

6b

\begin{tabular}{|c|c|c|c|c|c|c|c|}
\hline Entry & Letris acid & Temp. $\left({ }^{\circ} \mathrm{C}\right)$ & Tíme (h) & Yisld $(\%)$ & Endo: Exo's & Endo $\mathrm{ds}^{h}$ & Contig. \\
\hline 1 & morle & R'l' & 72 & 25 & $68: 32$ & 1.1 & $\$$ \\
\hline 2 & AlCly & 0 & 15 & 85 & $75: 25$ & 11 & $S$ \\
\hline 4 & $\mathrm{IiCl}_{4}$ & 0 & 17 & 77 & $66: 34$ & 16 & $\mathrm{~S}$ \\
\hline 5 & 'li(C)Pr' & R'l & 28 & 70 & $70: 30$ & 14 & $\mathrm{~S}$ \\
\hline
\end{tabular}

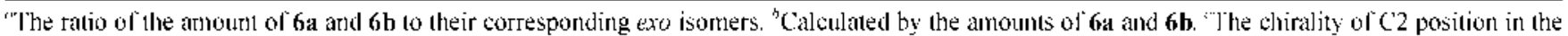
norbonene moicty of the major compound. "[he authentic 6 b was prepared from endo-(2 $R)-5$-norbonenc-2-carhoxylic acid."

compound, regardless of Lewis acids (entries 2-5).

$\left[\mathrm{iCl}_{\mathrm{f}}\right.$, a divalent metal compound. forms chelation with amide 3 and the ester carbonyl moiety, resulting that a cisoid conformation was preferred to the transoid one. On the other hand. $\mathrm{AlCl}_{3}$, a monovalent one, coordinated only by amide carbonyl group, resulting that the ransoid one was favored. ${ }^{6-8}$ As expected, a metal coordinated with the amide carbonyl group of compound 5 , and the same diastereomer was given as a Inajor compound.<smiles>C=CC(=O)N1C2CCC1C(C(=O)OCc1ccccc1)C(C(=O)OC)C2</smiles>

transoid

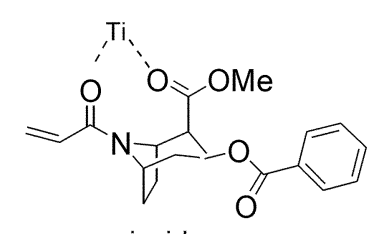

cisoid<smiles>C=CC(=O)N1C(CO)C2CCC1C(COC(=O)c1ccccc1)C2</smiles>

In Diels-Alder reaction, 8-membered chelation was once reported, but 8- or larger membered ring complex was often considered in other reactions, such as asymmetric hydrogenation." allylic alkylation." ${ }^{12}$ and so on. ${ }^{13}$ In summary, Lewis acids apparently affected the diastereofacial selectivity. A divalent Lewis acid might be involved in the formation of 8 -menbered chelation which was effective enough to distinguish between the transoid and cisoid comformer of a $N$-acryloyl chiral dienophile in Diels-Alder reaction with cyclopentadiene.

\section{Experimental Section}

All chemicals were reagent grade (Aldrich Chemical Co.) and were used as purchased without further purification. ${ }^{\mathrm{H}} \mathrm{H}$ and ${ }^{1 .} \mathrm{C}$ NMR spectra were recorded on a Varian Gemini
$2000(200 \mathrm{MHz})$ spectrometer in $\mathrm{CDCl}_{\overline{.}}$. Column chromatographic purifications were performed using 70-230 mesh silica gel.

Methyl $N$-acryl-8-aza-3 $\beta$-benzoyloxybicyclo|3.2.1|octane$2 \beta$-carboxylate (3). To a mixture of methyl 8 -aza- $3 \beta$ benzoyloxybicyclo[3.2.1] octane-2 $\beta$-carboxylate $(0.20 \mathrm{~g}$. $0.69 \mathrm{mmol}){ }^{1+}$ dichloromethane $(5 \mathrm{~mL})$, and triethylamine $(0.084 \mathrm{~g}, 0.83 \mathrm{mmol})$ was added dropwise acryloyl chloride $(0.087 \mathrm{~g}, 0.97 \mathrm{mmol})$ at $0{ }^{\circ} \mathrm{C}$. After stirring for $2 \mathrm{~h}$ at $\mathrm{rt}$, the mixture was quenched with $0.1 \mathrm{~N} \mathrm{HCl}$. The organic layer was washed with water and brine, dried over $\mathrm{MgSO}_{11}$, and concentrated in vacto. The oily residue was purified by column chromatography on silica gel (EtOAc/ hexane, $1: 1$ ) to give a colorless oil $(0.23 \mathrm{~g}, 97 \%)$. ' $\mathrm{H}$ NMR $\delta 1.82-2.14$ $(\mathrm{m}, 5 \mathrm{H}), 2.39-2.50(\mathrm{~m}, \mathrm{IH}), 3.08-3.16(\mathrm{~m}, 1 \mathrm{H}), 3.55,3.60(\mathrm{~s}$. 3H), 4.43-4.5I (m, IH), 4.90-5.07(m, IH), 5.40-5.5I (m. lH), 5.58-5.66 (m, IH), 6.22-6.25 (m, 2H), 7.30-7.35 (m. $3 \mathrm{H}), 7.86-7.90(\mathrm{~m}, 2 \mathrm{H}) ;{ }^{13} \mathrm{C}$ NMR $\delta 26.5,28.7,34.8,49.1$. $51.0,53.7,55.7,66.5,127.4,127.8,128.0,129.8,129.9$. $133.4,161.5,165.9,170.1$; HRMS found $m / z 344.0759$ $\left(\mathrm{MH}^{\circ}\right), \mathrm{C}_{10} \mathrm{H}_{22} \mathrm{NO}_{\text {s }}$ requires $344.3 \% 02$.

Methyl $N$-acryl-8-aza-3 $\beta$-benzoyloxybicyclo|3.2.1 |octane$2 \alpha$-carboxylate (5). Compound 5 was prepared from methyl 8-aza-3 $\beta$-benzoyloxybicyclo[3.2.I ] octane- $2 \alpha$-carboxylate ${ }^{14}$ by the above mentioned method. 'H NMR $\delta$ I.80-2.50 (m. $6 \mathrm{H}), 2.85-3.18(\mathrm{~m}, 1 \mathrm{H}), 3.59(\mathrm{~s}, 3 \mathrm{H}), 4.28-4.60(\mathrm{~m}, \mathrm{IH})$. 4.70-4.96 (m, 1H), 5.60-5.80 (m, 2H), 6.26-6.40 (m, 2H), $7.22-7.54(\mathrm{~m}, 3 \mathrm{H}), 7.86-7.98(\mathrm{~m}, 2 \mathrm{H}) ;{ }^{1 .} \mathrm{C}$ NMR $\delta 28.9$. $29.8,38.0,50.6,53.5,54.1,55.8,67.7,127.5,128.5,129.2$. $129.8,130.0,133.3,163.0,165.7,171.2$.

General procedure for Diels-Alder reaction. To a stirted solution of $3(0.050 \mathrm{~g}, 0.122 \mathrm{mmol})$ in dichloromethane $(1.5$ $\mathrm{mL}$ ) was added a solution of 2 equiv. Lewis acid in dichloromethane $(5 \mathrm{~mL})$ at $0^{\circ} \mathrm{C}$. After stitring for $30 \mathrm{~min}$, a solution of cyclopentadiene $(0.04 \mathrm{~g} .0 .61 \mathrm{mmol})$ in dichloromethane $(5 \mathrm{~mL}$ ) was added during $10 \mathrm{~h}$ with a syringe pump at the respective temperature. The mixture was stirred for additional $5 \mathrm{~h}$ and then quenched with $0.1 \mathrm{~N} \mathrm{HCl}$. The organic layer was washed with water and brine, dried over $\mathrm{MgSO}_{\mathrm{s}}$, and concentrated in vacuo. The oily residue was 
purified by column chromatography on silica gel (EtOAc/ hexane, $1: 3$ ). and the yield and the diastereomeric ratio were determined by HPLC analysis. ${ }^{7}$

4b: ${ }^{1} \mathrm{H}$ NMR $\delta$ 1.24-1.49 (m, 4H), 1.73-2.11 (m. 5H). 2.65-3.17 (m. 5H). 3.65. 3.67 (s. 3H). 4.58-4.75 (m. lH). 4.86-5.06 (m. IH) $5.46-5.52$ (m. lH). 5.77-6.24 (m. $2 \mathrm{H})$. 7.34-7.52 (m. 3H). 7.87-7.95 (m. 2H); ${ }^{13} \mathrm{C}$ NMR $\delta 27.0$. $29.8,30.2,34.9,41.9,42.8,46.5,48.7,49.9,52.0 .53 .0 .53 .2$. 66.8, 128.6. 129.8, 130.0. 133.0, 133.4. 136.5. 166.0, 169.9. 170.3: HRMS found $m z+10.1967\left(\mathrm{MH}^{-}\right), \mathrm{C}_{24} \mathrm{H}_{z} \sharp \mathrm{NO}_{s}$ requires 410.1969 .

6b: ${ }^{1} \mathrm{H}$ NMR $\delta 1.29-1.69(\mathrm{~m}, 4 \mathrm{H}), 1.75-2.33$ (m. $5 \mathrm{H}$ ). 2.91-3.30 (m. $5 \mathrm{H}), 3.62(\mathrm{~s}, 3 \mathrm{H}), 4.40-4.64(\mathrm{~m}, \mathrm{lH}) .4 .67$. $4.90(\mathrm{~m} . \mathrm{lH}), 5.63-5.76(\mathrm{~m} . \mathrm{lH}) .5 .97-6.10(\mathrm{~m}, \mathrm{lH}), 6.17$. $6.21(\mathrm{~m}, \mathrm{lH}) .7 .35-7.56(\mathrm{~m} .3 \mathrm{H}), 7.92-7.96(\mathrm{~m}, 2 \mathrm{H}) ;{ }^{13} \mathrm{C}$ NMR $\delta 27.0 .29 .8,30.2 .31 .2,42.9 .46 .5,49.9 .50 .4,51.2$. $52.6,53.2,55.8,67.8,128.5,129.8,130.0 .132 .5,133.2$. $138.3,165.7,170.9,171.3$

Acknowledgment. We are grateful for the support of this work through a grant from National Research Laboratory program.

\section{References}

1. Carruther. W. Cycloaddition Reactions in Organic Synthesis' Pergamon Press: Oxford. 1990

2. Seydens-Pe. J. Chiral Altxifiaries and Ligands in Asnmtetric Smmesis: John Wiley \& Sons. Inc: New York. 1995.

3. Corminboeuf. O.: Renaud. P. Org. Lett. 2002, 1,1735

4. Ruck-Braum. K: Kunz. H. Chiral Atwiliaries in Cycloaddition: Wiley-VCH: New York, 1999.

5. Oppolzer. W. Angew: Chem, Int Ed Engl 1984. 23.876.

6. Waldmann. H. J. Org Chent 1988.53.6133

7. Park. D. H.: Kim. S. H.: Kim. T. D.: Kim. Y.H. Chem. Conmum. 1999. 963

8. Chung. K.-H.: Chu, C.-K.: Chang, M.-H. Heterocycles 2003. 60. 2141.

9. Poll. T.: Metter. T. O.: Helmehet1. G. Angew: Chem 1985. 97. 116.

10. (a) Castellino. S.: Divight. W. I. J. Ant Chent. Soc. 1993. 115. 2986. (b) Evans. D. A.: Chapman. K. T.: Bisaha. I. T. J.Am. Chent Soc. $1988,110,1238$.

11. Morrison. J. D. Adv Catal 1976. 25,81.

12. (a) Hayashi. T; Kanehira. K.: Tsuchiya. H.: Kumada. M. J. Chem. Soc. Chem. Conmm. 1982. 1162 . (b) Trost. B. M.: Murphy. D. J. J. Onganomet. 1985. +. 1143 .

13. Tamao. K.: Yamamoto. H.: Myake. N.: Hayashi. T.: Kumada. M. Tetrahedhon Lett. 1977, 1389.

14. Singh. S. Chem. Rev 2000. $100,925$. 